\title{
Structural and Functional Denervation of Human Detrusor after Spinal Cord Injury
}

\author{
Marcus J. Drake, Petter Hedlund, Ian W. Mills, Rachel McCoy, Gordon McMurray, \\ Brian P. Gardner, Karl-Erik Andersson, and Alison F. Brading
}

Oxford Continence Group (MJD, IWM, RM, GM, AFB), University Department of Pharmacology, Oxford, and Urology Department (MJD), Churchill Hospital, Oxford, United Kingdom; and Department of Clinical Pharmacology (PH, K-EA), Lund University Hospital, Lund, Sweden; and National Spinal Injury Centre (BPG), Stoke Mandeville, Aylesbury, United Kingdom

\begin{abstract}
SUMIMARY: The bladder receives an extensive nerve supply that is predominantly cholinergic, but several putative transmitters are present, some of which are colocalized. Previous studies have shown increased levels of sensory nerves, reduced inhibitory transmitters, and structural and functional changes in the excitatory input in unstable bladder conditions. The present study compared the end-organ nerve supply to the bladder in spinal cord injury (SCl) with uninjured controls. Acetylcholinesterase histochemistry and double-label immunofluorescence were used to investigate neurotransmitter content, with confocal laser scanning microscopy to assess colocalization. Organ bath studies provided functional correlates for the structural changes in the excitatory innervation. Control samples had dense innervation of the detrusor containing a diverse range of transmitters. Hyperreflexic SCl samples showed patchy denervation, and areflexic SCl samples were diffusely denervated. Vasoactive intestinal polypeptide-, neuropeptide $\mathrm{Y}$-, neuronal nitric oxide synthase-, and galanin-immunoreactive nerve fibers were reduced from frequent or moderately frequent to infrequent or very infrequent in SCl. Calcitonin gene-related peptide-immunoreactive fibers were infrequent in controls and $\mathrm{SCl}$ samples. Patterns of colocalization were unchanged, but significantly fewer fibers expressed more than one transmitter. The subepithelial plexus was markedly reduced and several of the smaller coarse nerve trunks showed no immunoreactivity to the transmitters assessed. There was no reduction in sensitivity to electrical field stimulation of intrinsic nerves in $\mathrm{SCl}$, but the maximum force generated by each milligram of bladder tissue and the peak force as a proportion of the maximum carbachol contraction were significantly reduced and the responses were protracted. There was no significant functional atropine-resistant neuromuscular transmission in controls or SCl. The reported findings have clinical implications in the management of chronic SCl and development of new treatments. (Lab Invest 2000, 80:1491-1499).
\end{abstract}

$D$ etrusor muscle receives an extensive nerve supply (Daniel et al, 1983), and there is a dense suburothelial plexus in the bladder (Gosling et al, 1983). Excitatory neuromuscular transmission in the normal human bladder is predominantly cholinergic (Brindley and Craggs, 1976; Chen et al, 1994; Kinder and Mundy, 1985; Sibley, 1984; Sjogren et al, 1982), but a wide range of putative transmitters is present. These include nitric oxide (NO) (Smet et al, 1994), vasoactive intestinal polypeptide (VIP), calcitonin gene-related peptide (CGRP), and tachykinins (Smet et al, 1997). Several of these have been shown to immunolocalize within the same nerve fiber on doublelabeling studies (Smet et al, 1997), and it is possible that they serve as modulators of neuromuscular trans-

\section{Received March 10, 2000}

This work was supported by the Royal College of Surgeons of England, London, United Kingdom; by the Lund/Oxford Biomedical Research Exchange Scheme, Lund, Sweden; and by charitable funds of the National Spinal Injury Centre, Stoke Mandeville and Radcliffe Hospitals NHS Trust, Oxford, United Kingdom.

Address reprint requests to: Dr. Marcus Drake, Department of Surgery, School of Surgical Sciences, The Medical School, University of Newcastle, Newcastle upon Tyne, NE2 4HH, United Kingdom. Fax: 44191222 8514;E-mail:m.j.drake@ncl.ac.uk mission and smooth muscle activity. Intramural ganglia are present in the lateral bladder wall and are heterogeneous in respect of VIP-, neuronal NO synthase- (n-NOS), neuropeptide Y (NPY)-, tyrosine hydroxylase (TH)-, and galanin (gal)-immunoreactivity (IR) (Smet et al, 1996). Pericellular varicosities, most often expressing CGRP- or VIP-IR, occur around ganglion cells (Smet et al, 1996). The arrangement suggests the possibility of local circuits regulating or reinforcing motor or sensory activity. Nevertheless, considerable uncertainty remains regarding the origin of terminal motor and sensory fibers and the precise function of the substances they contain.

Some studies into pathological causes of unstable bladder activity have looked for an increase in the number of sensory nerve fibers, hypothesizing that detrusor instability (DI) may result from abnormal afferent activity in the micturition reflex. Increased numbers of nerves containing acetylcholinesterase (AChE) (Moore et al, 1992), CGRP (Smet et al, 1997), and substance P (SP) (Smet et al, 1997) are present in the suburothelial region. However, there is no alteration in the overall suburothelial nerve density (Smet et al, 1997). Changes in detrusor innervation occur, with reduced densities of some transmitters in hyperreflexia (Kinder et al, 1985), idiopathic DI (Gu et al, 1983), 
and bladder outflow obstruction (Chapple et al, 1992), and alterations in cholinergic nerve distribution in idiopathic DI (Charlton et al, 1999; Mills et al, 2000). The morphological studies have been supported by functional experiments. Partial denervation and cholinergic supersensitivity is evident in isolated perfused strips from people with unstable obstructed bladders (Harrison et al, 1987) or hyperreflexia due to spina bifida (German et al, 1995). In idiopathic DI there is reduced response to electrical field stimulation of intrinsic nerves (Mills et al, 2000) and increased spontaneous activity (Kinder and Mundy, 1987; Mills et al, 2000), but these are not associated with cholinergic supersensitivity (Mills et al, 2000). Ultrastructurally, elderly patients with idiopathic DI show detrusor denervation, seen as axon degeneration, associated with a specific muscle dysjunction pattern (Elbadawi et al, 1993).

Spinal cord injury (SCl) has fundamental effects on bladder function, usually resulting in overactivity (hyperreflexia) in suprasacral lesions or inability to void (areflexia) in more distal lesions. Since the clinical manifestations of detrusor hyperreflexia are similar to those of idiopathic and obstructive DI, there may be common pathophysiology (Brading and Turner, 1994). Nevertheless, the different nomenclature for the clinical conditions has been proposed by the Standardization Committee of the International Continence Society (Abrams et al, 1986). SCl is of particular interest in that most people affected have achieved full maturity and normal bladder function before the injury, contrasting with the congenital neuropathic diseases that have been the subject of previous investigations into hyperreflexia (German et al, 1995; Kinder and Mundy, 1987; Saito et al, 1993). In the current study we aimed to investigate how $\mathrm{SCl}$ affects end-organ innervation in the bladder in terms of function, structure, and neurotransmitter expression and colocalization.

\section{Results}

\section{Histochemistry, Immunofluorescence, and Confocal Microscopy}

Detrusor Muscle. Controls showed very frequent AChE-positive nerves (Fig. 1) and very frequent fibers that are immunoreactive to the general nerve marker protein gene product (PGP) 9.5. These markers revealed slender nerve fibers and varicose terminals evenly distributed throughout the detrusor muscle. There were frequent VIP-immunoreactive varicose terminals and moderate numbers of fine varicose terminals with NPY-, n-NOS-, and gal-IR, infrequent CGRP$I R$, and very infrequent TH- and SP-IR. TH-IR was more apparent in interfascicular planes. Hemoxygenase (HO)-1, HO-2, and somatostatin (SOM)-IR were absent. Colocalization was apparent between several transmitters (Fig. 2) and the associations are set out in Table 1.

$\mathrm{SCl}$ samples showed marked reduction in the number of nerve fibers in the detrusor using both AChE histochemistry and PGP 9.5 immunofluorescence. The pattern of denervation was patchy in hyperreflexic $\mathrm{SCl}$, in which some areas showed frequent nerve fibers adjacent to areas where nerve fibers were very infrequent (Fig. 1). Nerve loss was diffuse in areflexic $\mathrm{SCl}$, down to very infrequent (absent in one specimen) (Fig. 1). VIP-, NPY-, n-NOS, and gal-IR were reduced to infrequent or very infrequent. CGRP-IR was infrequent in controls and remained infrequent in $\mathrm{SCl}$ samples. TH- and SP-IR were almost absent from the muscle fascicles. There was no change in the patterns of colocalization assessed, but significantly fewer fi-

1 a)

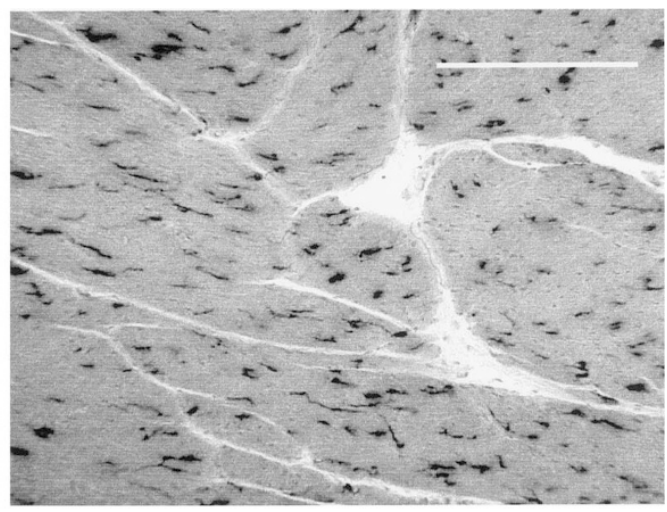

$1 \mathrm{~b})$

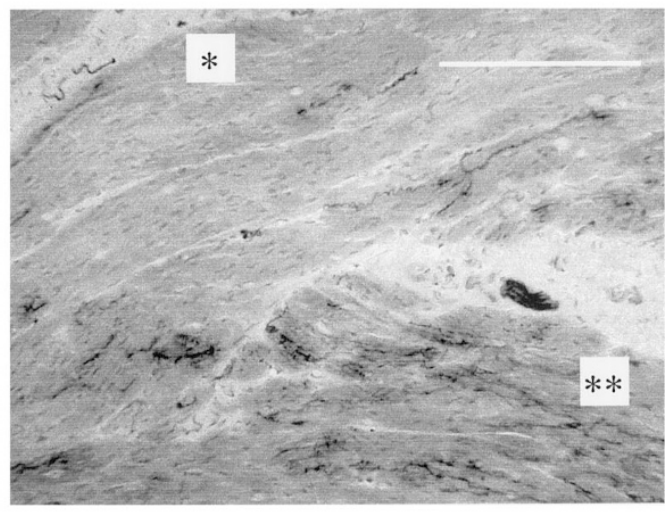

$1 \mathrm{c)}$

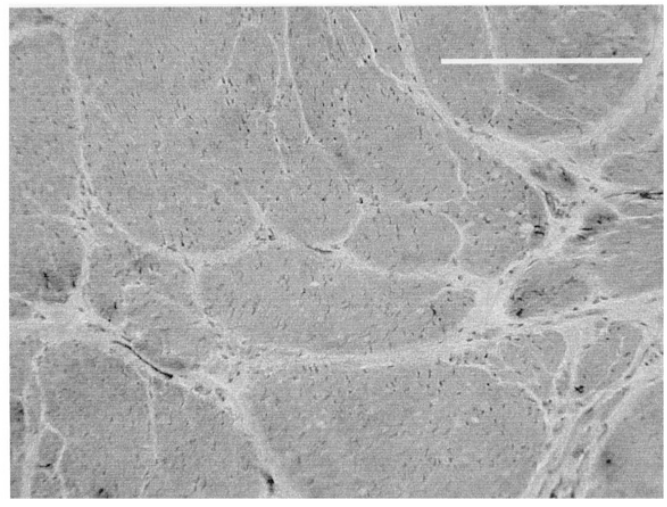

Figure 1.

Detrusor muscle innervation. a, Control (44-year-old female cadaveric organ donor). Numerous black deposits of reaction product indicative of presumptive cholinergic nerves. $b$, Patchy denervation (33-year-old female hyperreflexic spinal cord injury [SCI]). Denervated fascicle $\left({ }^{*}\right)$ adjacent to a well-innervated fascicle $\left.{ }^{* \star}\right) . c$, Diffuse denervation (48-year-old male areflexic SCI). Near-total detrusor denervation. A few nerve fibers are present in interfascicular planes. Acetylcholinesterase histochemistry. Scale bar $=100 \mu \mathrm{m}$. 
a)

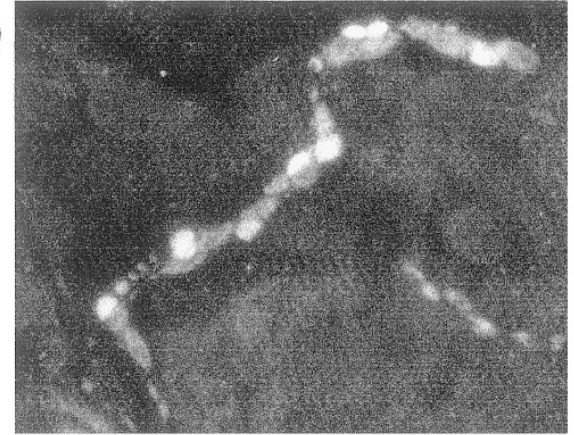

b)

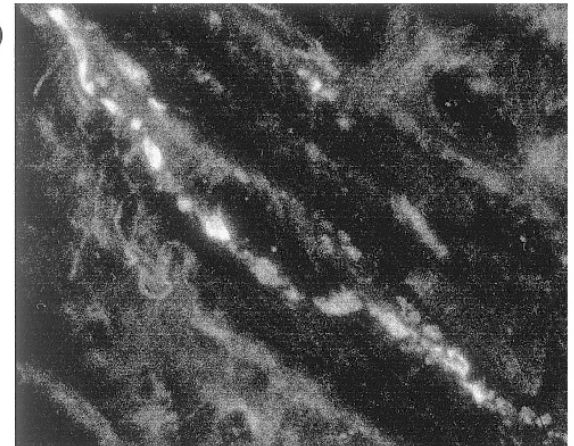

c)

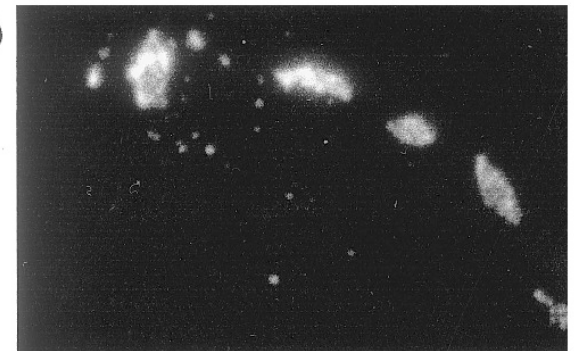

d)

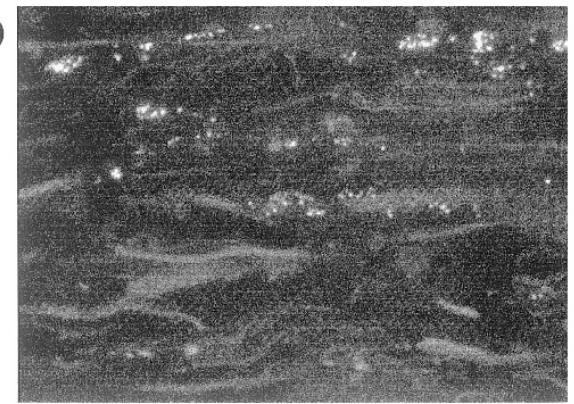

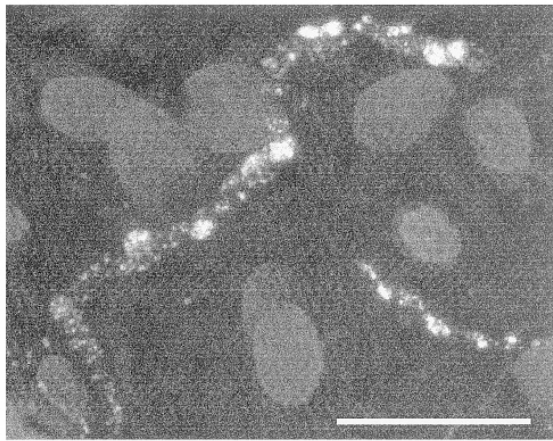
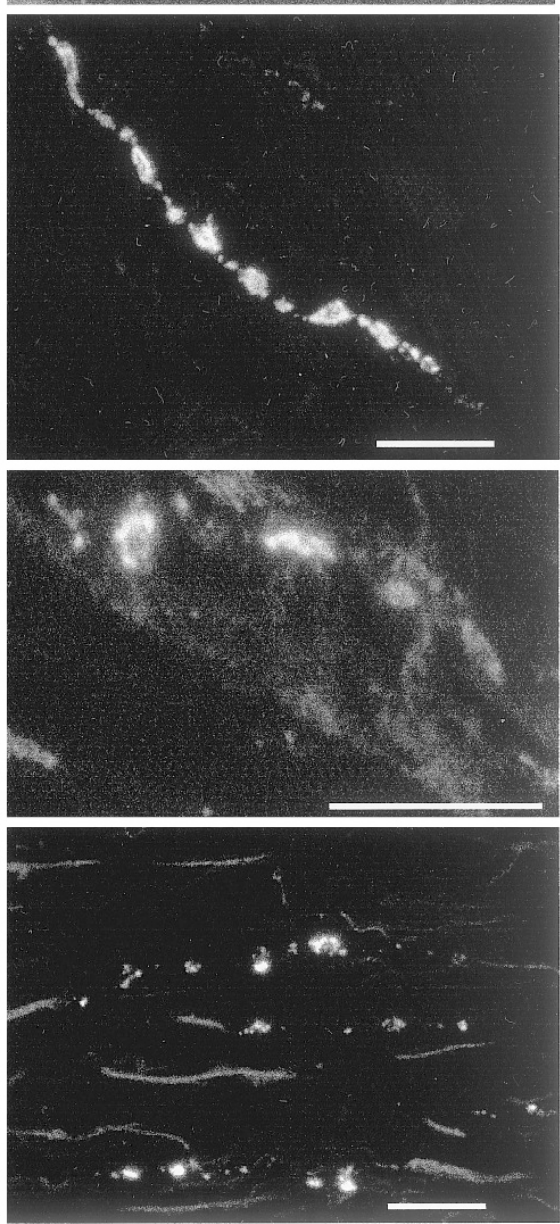

\section{Figure 2.}

Neurotransmitter colocalization. a, 33-year-old control; n-NOS (left) colocalized with vasoactive intestinal polypeptide (VIP) (right). b, 47-year-old areflexic SCl; n-NOS (left) colocalized with VIP (right). c, 33-year-old control; calcitonin gene-related peptide (CGRP) (left) colocalized with galanin (gal) (right). $d$, 47-year-old areflexic SCl: CGRP (left) close to but not colocalized with gal (right). Scale bar $=10 \mu \mathrm{m}$. Double-label immunofluorescence viewed by confocal microscopy.

bers expressed more than one transmitter $(p<0.01)$ (Table 1).

Suburothelial Plexus. Controls showed an extensive suburothelial nerve plexus, with very frequent AChEpositive and PGP 9.5 immunoreactive fibers (Fig. 3), from which nerve fibers extended into the urothelium. Innervation was frequent for VIP-IR, moderate for NPY-, SP-, TH-, CGRP-, and n-NOS-IR, and infrequent for gal- and SOM-IR. HO-1-IR was absent. In $\mathrm{SCI}$ the subepithelial plexus was markedly reduced; in some areas it was absent, but changes in hyperreflexic cases were akin to those seen in fascicles in that some areas showed preservation of the plexus at a reduced density (Fig. 3). Colocalization associations (Table 1) were the same as for controls, but significantly fewer fibers expressed more than one transmitter $(p<0.05)$ (Table 1$)$.

Other Regions. Coarse nerve trunks were seen in interfascicular planes in the detrusor, taking up a characteristic "wavy" conformation. Large nerve trunks in controls showed VIP-, CGRP-, NPY-, TH-, and SP-IR. Nearly all of the smaller trunks contained VIP-IR, but they often lacked one or more of the other transmitters. In SCI, VIP-IR remained the commonest finding, but some smaller trunks were devoid of VIP$\mathrm{IR}$, with several showing no IR to the transmitters 
Table 1. Colocalization of transmitters in nerve fibers in controls and SCI as assessed with confocal laser scanning microscopy

\begin{tabular}{|c|c|c|c|c|c|c|c|c|}
\hline \multirow[b]{2}{*}{ Transmitter } & \multirow{2}{*}{$\begin{array}{l}\text { Nerve site } \\
\text { (frequency) }^{a}\end{array}$} & \multicolumn{7}{|c|}{ Proportion colocalizing second transmitter (control/SCI) ${ }^{b}$} \\
\hline & & VIP & NPY & CGRP & n-NOS & TH & Gal & SP \\
\hline \multirow[t]{2}{*}{ VIP } & Muscle (F/l) & - & $4 / 3$ & $0 / 0$ & $2 / 1$ & NA & NA & $0 / 0$ \\
\hline & Suburo. ${ }^{c}(F / I)$ & - & $4 / 4$ & $0 / 0$ & $2 / 1$ & & & $0 / 0$ \\
\hline \multirow[t]{2}{*}{ NPY } & Muscle (M/I) & $4 / 2$ & - & $1 / 0$ & NA & $3 / 1$ & NA & NA \\
\hline & Suburo. (M/VI) & $4 / 3$ & - & $2 / 2$ & & $1 / 1$ & & \\
\hline \multirow[t]{2}{*}{ CGRP } & Muscle $(\mathrm{I} / \mathrm{I})$ & $0 / 0$ & $2 / 0$ & - & NA & NA & $3 / 1$ & $2 / 1$ \\
\hline & Suburo. (M/l) & $0 / 0$ & $2 / 1$ & - & & & $3 / 1$ & $2 / 1$ \\
\hline \multirow[t]{2}{*}{ n-NOS } & Muscle (M/VI) & $3 / 2$ & NA & NA & - & $\mathrm{CA} / 0$ & NA & NA \\
\hline & Suburo. (M/l) & $3 / 2$ & & & - & $0 / 0$ & & \\
\hline \multirow[t]{2}{*}{ TH } & Muscle (VI/A) & NA & $4 / 1$ & NA & $\mathrm{CA} / 0$ & - & NA & NA \\
\hline & Suburo. (M/VI) & & $4 / 1$ & & $0 / 0$ & - & & \\
\hline \multirow[t]{2}{*}{ Gal } & Muscle (M/VI) & NA & NA & $2 / 1$ & NA & NA & - & NA \\
\hline & Suburo. (I/VI) & & & $2 / 1$ & & & - & \\
\hline \multirow[t]{2}{*}{ SP } & Muscle (VI/A) & $0 / 0$ & NA & $5 / 5$ & NA & NA & NA & - \\
\hline & Suburo. (M/I) & $0 / 0$ & & $5 / 5$ & & & - & \\
\hline
\end{tabular}

${ }^{a} \mathrm{~A}=$ Absent; $\mathrm{VI}=$ Very infrequent; I = Infrequent; $\mathrm{M}=$ Moderate; $\mathrm{F}=$ Frequent; $\mathrm{VF}=$ Very frequent; $(\mathrm{Control} / \mathrm{SCI})$

${ }^{b} 0=$ No colocalization present; $1=1-20 \% ; 2=21-40 \% ; 3=41-60 \% ; 4=61-80 \% ; 5=81-100 \%$; CA = close association observed, but transmitters not colocalized; NA = not assessed.

${ }^{c}$ Suburo $=$ suburothelial plexus

assessed. Intramural ganglia were very infrequent in the dome of the bladder for both controls and SCl. Fine nerve fibers were observed around $86 \%$ of arteries in controls stained for PGP 9.5. They showed frequent TH-, NPY-, and VIP-IR, with moderate or infrequent SP- and CGRP-IR. In SCI, perivascular fibers were seen for $47 \%$ of arteries. The same transmitters were present as for controls, but with a proportionate reduction in the frequency of the nerves showing IR.

Functional Studies. The numbers of strips studied were: controls 127 (mean weight $3.7 \mathrm{mg}$ ), hyperreflexic SCI 108 (3.4 mg), and areflexic SCI 92 (3.5 mg). Electrical field stimulation (EFS) caused a rapid rise in isometric tension that was frequency-dependent in the range 1 to $50 \mathrm{~Hz}$ (Fig. 4). The time course of EFS responses in the $\mathrm{SCl}$ strips was significantly altered. The time to peak response for controls (expressed as mean \pm SEM) was $5.8 \pm 0.4$ seconds, compared with $12.8 \pm 0.9$ seconds for hyperreflexic $\mathrm{SCl}$ and $11.2 \pm$ 0.5 seconds for areflexic SCI $(p<0.01)$. The $50 \%$ to $50 \%$ contraction time for controls was $14.4 \pm 0.7$ seconds, compared with $26.9 \pm 2.5$ seconds for hyperreflexic $\mathrm{SCl}$ and $22.1 \pm 2.1$ seconds for areflexic $\mathrm{SCl}(p<0.01)$. There was no reduction in the sensitivity to EFS in SCl (Fig. 5a). However, the maximum force generated by each milligram of bladder tissue was significantly reduced (Fig. $5 \mathrm{~b}$ ) at $0.61 \pm 0.06 \mathrm{~g} / \mathrm{mg}$ for hyperreflexic cases and $0.43 \pm 0.07 \mathrm{~g} / \mathrm{mg}$ for areflexic, compared with $1.37 \pm 0.09 \mathrm{~g} / \mathrm{mg}$ in controls $(p<0.01)$. The peak EFS response as a proportion of the maximum carbachol contraction (Fig. 5c) was 72.1 $\pm 3.6 \%$ for controls, $62.3 \pm 2.8 \%$ for hyperreflexic $\mathrm{SCl}$, and $51.2 \pm 2.5 \%$ for areflexic $\mathrm{SCl}(p<0.01)$. In the control strips, $13.6 \pm 4.1 \%$ of the EFS response at $30 \mathrm{~Hz}$ was atropine resistant, compared with $11.7 \pm$ $3.1 \%$ and $11.1 \pm 3.3 \%$ for hyperreflexic and areflexic
$\mathrm{SCl}$, respectively $(p=0.23)$. Additional perfusion with tetrodotoxin further reduced the responses for control strips to $10.1 \pm 2.9 \%$, for hyperreflexic SCl strips to $8.9 \pm 2.4 \%$, and for areflexic strips to $8.7 \pm 3.2 \%(p=$ $0.69)$.

\section{Discussion}

The major findings in the reported study relate to detrusor and suburothelial denervation in SCl. Hyperreflexic cases were distinguished by the patchiness of the denervation, particularly in the detrusor and to a lesser extent in the suburothelial plexus. Areflexic cases showed diffuse, severe nerve loss. The reduction was global for all tested transmitters and appeared to be a loss of both the slender fibers and varicose terminals rather than merely loss of the IR. Detrusor denervation occurs in humans with congenital neuropathic hyperreflexia (Charlton et al, 1999; German et al, 1995), idiopathic DI (Charlton et al, 1999; Mills et al, 2000), and obstructive DI (Harrison et al, 1987; Speakman et al, 1987). In some, the denervation has been reported as patchy, akin to the current findings (Charlton et al, 1999; Mills et al, 2000). In hyperreflexic $\mathrm{SCl}$, the apparent density in areas retaining innervation was reduced compared with agematched controls. This suggests that the denervation in hyperreflexic cases may be a combination of two processes, one leading to a patchy change, on which is superimposed a diffuse denervation. The diffuse nerve loss in areflexic $\mathrm{SCl}$ cases is similar to a severe version of the nerve loss seen with ageing in normal humans (Gilpin et al, 1986). We postulate, therefore, that SCI leads to a general acceleration of the ageing process, either directly, indirectly due to obstruction from dyssynergic sphincter activity, or as a consequence of the increased functional demands placed 

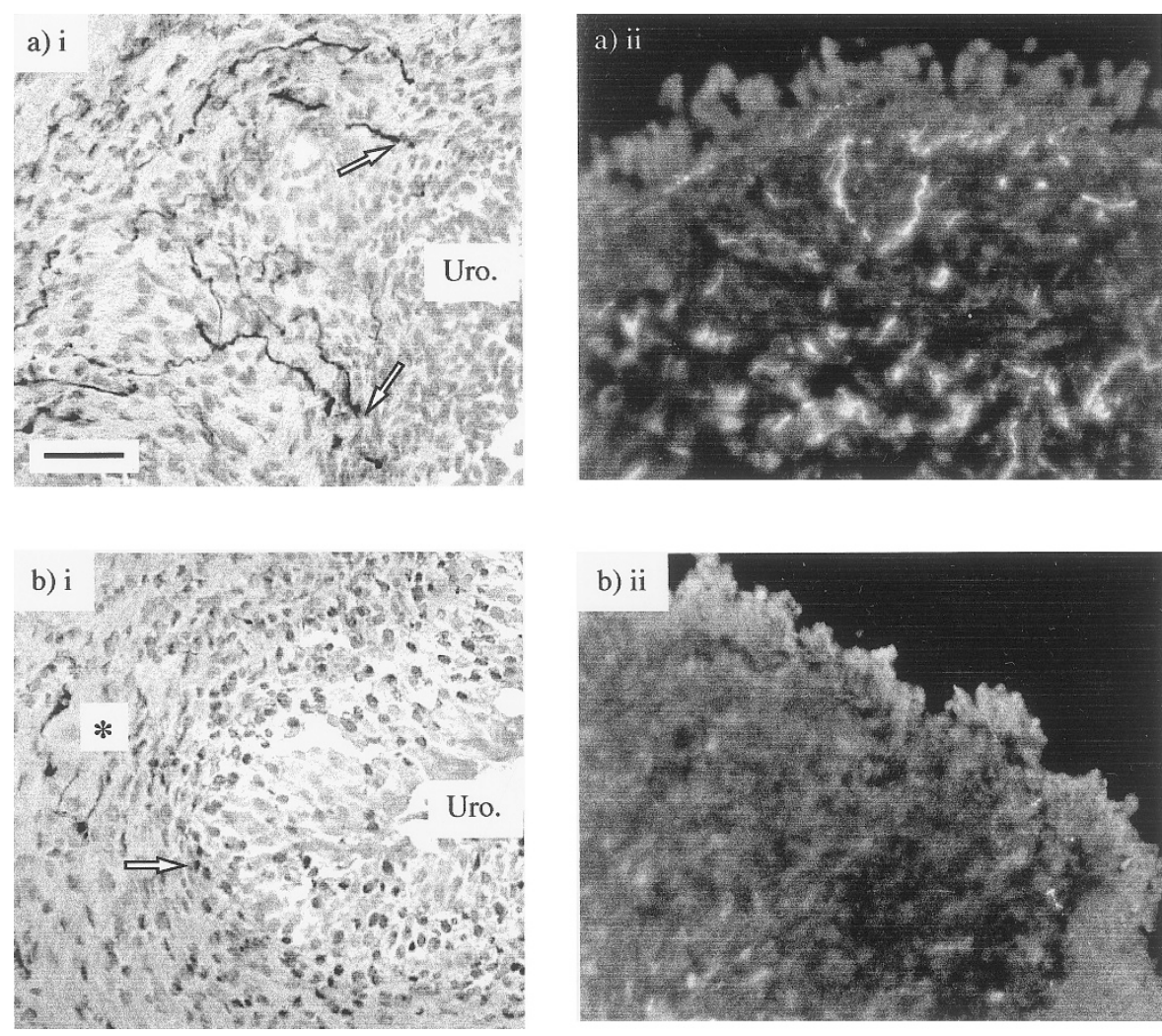

\section{Figure 3.}

Suburothelial plexus. $a(\lambda)$, Control (44-year-old male cadaveric organ donor). Suburothelial nerve fibers (arrowhead) were seen approaching the urothelial basement membrane. Acetylcholinesterase histochemistry. a (ii), Section from the same control specimen. Protein gene product (PGP) 9.5 immunofluorescence. $b(i)$, SCl (33-year-old male hyperreflexic). Marked suburothelial nerve loss, with only sporadic fibers still seen $\left({ }^{\star}\right)$. Arrowhead indicates region of urothelial basement membrane. Acetylcholinesterase histochemistry. $b$ (ii), Section from the same SCl specimen showing an area of total denervation. PGP 9.5 immunofluorescence. Uro. $=$ urothelium; scale bar $=10 \mu \mathrm{m}$ (applies to all).

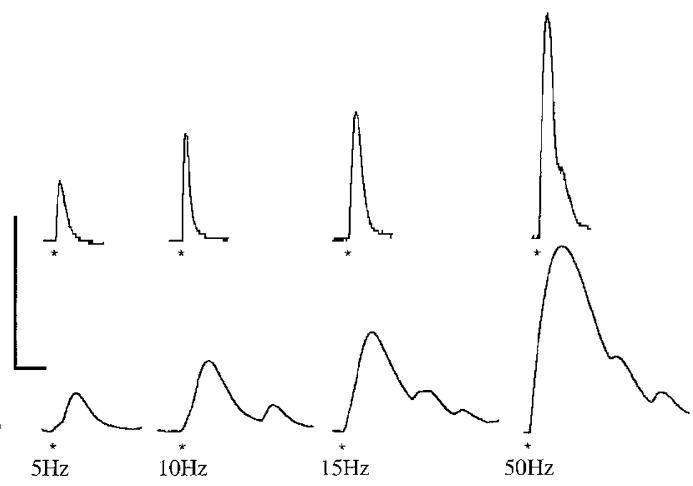

Figure 4.

Example electrical field stimulation (EFS) traces. a, Control (42-year-old female, strip weight $2.1 \mathrm{mg}$ ). $b, \mathrm{SCl}$ (44-year-old male hyperreflexic, strip weight $3.9 \mathrm{mg}$ ). Protracted, multiphasic response and reduced force generation per milligram of tissue. Scale: vertical bar, $1 \mathrm{mg}$ isometric tension; horizontal bar, 15 seconds. * indicates the point of stimulation.

on the injured individual (Whiteneck et al, 1993). Despite denervation of a large proportion of the muscle in hyperreflexic SCl specimens, the ability to achieve detrusor contraction in vivo is maintained. In normal bladder, muscle innervation is particularly dense (Daniel et al, 1983) and may be surplus to the minimum required for bladder emptying, potentially providing an innervation reserve. Alternatively, changes in detrusor properties, such as increased smooth muscle coupling, may compensate and thereby facilitate widespread contraction after partial denervation.

The etiology of the nerve loss is not apparent, but all parts of the dome of the bladder were affected in the current study. The range of transmitters seen in the coarse nerve trunks was reduced, which suggests a proximal process, perhaps at the level of the pelvic ganglia. In view of the persisting immunolocalization of PGP 9.5 by coarse nerve trunks in $\mathrm{SCl}$ samples, a local process selectively affecting terminal parts of nerve fibers is also possible. There is evidence that transmural ischemia may explain denervation in idiopathic DI (Mills, 1999).

Failure to observe HO-1 and 2 in these experiments, and low levels of other transmitters, may not be a true reflection of their representation in the dome of the bladder. Nonvisualization could also occur if the transmitter is labile in vivo or suffers rapid depletion post mortem, a comment that applies to immunolocalization studies in general. HO-2 produces carbon monoxide (CO), a putative transmitter found in the human urethra (Ho et al, 1999). HO-1 is inducible in response to oxidative stress. HO-2 undergoes axonal transport so that the local concentration in axons may be insufficient to allow immunolocalization (Vollerthun et al, 1995). Notwithstanding, functional studies show a 

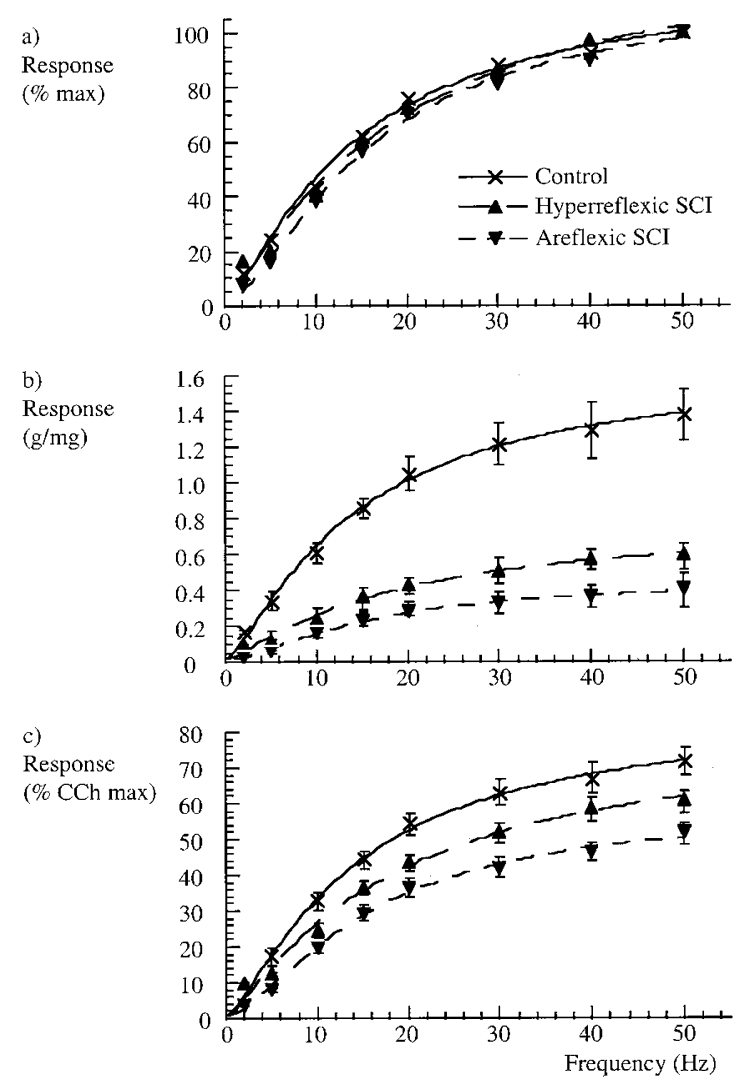

Figure 5.

Frequency-response curves of control and $\mathrm{SCI}$ detrusor strips to electrical field stimulation. Points are means \pm SEM. $a$, Contractile response of control $(n=$ 127), hyperreflexic $S C I(n=108)$, and areflexic $S C I(n=92)$ strips expressed as a percentage of the maximal response to EFS. $b$, Contractile response of strips expressed in grams per milligram of tissue. $c$, Contractile response of strips expressed as the percentage of the maximal response to carbachol (CCh).

lack of effect of $\mathrm{CO}$ on human intravesical ureter (Iselin et al, 1997), and because the status of $\mathrm{CO}$ as a neurotransmitter remains uncertain, lack of $\mathrm{HO}$ enzymes in the human bladder might be expected.

Colocalization of neurotransmitters has been reported in the human bladder on the basis of coincident profiles on immunolocalization studies. At least three distinct populations of nerves are present, showing IR to VIP alone, CGRP alone, and CGRP with SP or neurokinin A (Smet et al, 1997). Postnatally, intramural ganglion cells colocalize NOS and NPY, SP and CGRP, or NPY and VIP (Dixon et al, 1998). The functional significance of transmitter colocalization is not known. These substances may have a trophic function or modulate muscle excitability or gene expression. Consequently, transmitter depletion and reduced colocalization may play a role in the reduced compliance and structural derangement of the bladder wall in SCl. The current results show similar colocalization patterns for the detrusor and the suburothelial region, except that fewer NPY-IR fibers contained TH-IR suburothelially, whereas the same proportion of TH-positive fibers contained NPY-IR in both regions. This implies two populations of NPY-IR nerves, those with or without $\mathrm{TH}-\mathrm{IR}$, the latter occurring more commonly in the suburothelial plexus.

The reduced peak force generated by bladder strips in acquired spinal cord dysfunction due to $\mathrm{SCl}$ contrasts with increased contractility of strips in congenital neurogenic bladder dysfunction (Saito et al, 1993). The study group in the latter investigation was younger than the controls (mean 12.4 years as against 44.8 years), making direct comparison difficult. The reduction in the current study could in part be due to connective tissue infiltration decreasing the amount of muscle present in each strip, but the greater reduction in response to EFS as compared with carbachol stimulation implies partial functional denervation. The protracted time course of the EFS response suggests that the total force generated may be similar to controls. This might indicate abnormal spread of excitation in response to nerve activity, which is likely to be myogenic. No significant atropine-resistant neuromuscular transmission was present and in essence all the residual contraction was also resistant to tetrodotoxin, indicating that it was due to direct muscle stimulation rather than nerve stimulation and neurotransmitter release. Atropine virtually eliminates nerveinduced contraction in normal human bladder (Kinder and Mundy, 1985; Sjogren et al, 1982), but noncholinergic nonadrenergic (NANC) neuromuscular communication is present in several other species. Atropine-resistance in humans has been reported in some pathological conditions (Saito et al, 1993; Sjogren et al, 1982), but such findings could actually have been due to direct muscle stimulation. NANC neurotransmission may be more prevalent with lowfrequency EFS (Brading and Williams, 1990; Luheshi and Zar, 1990), with the cholinergic component predominating at higher frequencies. Although a relatively high frequency $(30 \mathrm{~Hz})$ was used for investigating atropine resistance, it could be assumed to achieve maximal release of any NANC neurotransmitter, such that muscarinic blockade should reveal any NANCmediated contraction. This is seen in strips from animals with a large NANC component, such as the rat and the guinea pig (Brading and Williams, 1990). Consequently, it is unlikely that any major NANCmediated contraction was missed.

The etiology of unstable detrusor activity remains to be explained. Unstable contractions may be due to abnormal muscle properties ("the myogenic hypothesis") (Brading and Turner, 1994). Alternatively, the contractions may follow abnormal activation of the micturition reflex ("neurogenic hypothesis") (de Groat, 1997). The presence of subepithelial denervation, although not excluding the possibility, suggests that hyperreflexic contractions in $\mathrm{SCl}$ may not be due to exaggerated afferent activity. Likewise, abnormally protracted contraction in response to intrinsic nerve stimulation supports a myogenic etiology.

The reported findings are of clinical importance in the context of current bladder management deficiencies. All methods currently available are potentially associated with considerable morbidity, and there is increasing recognition of the need for novel ap- 
proaches. In light of the extensive structural and functional changes demonstrated, the etiology of which remains to be determined, new approaches should only be developed with due consideration to the possibility of declining effectiveness with time should denervation prove progressive.

\section{Materials and Methods}

\section{Study Subjects}

Informed consent and local ethical committee approval were obtained. Eighteen people with $\mathrm{SCl}$ and recent urodynamic evaluation were studied; 11 had detrusor hyperreflexia ( 7 male, 4 female, mean age 41.4 years) and 7 had areflexia (4 male, 3 female, mean age 44.4 years). Samples were obtained at elective open operation. Control samples (10 female, 7 male, mean age 48.7 years) were obtained from 11 cadaveric organ donors and from macroscopically normal areas of the bladder of 6 carcinoma patients undergoing cystectomy ( 2 had previously received mitomycin intravesical chemotherapy). Urodynamic investigation was not possible for controls, but none had any history of lower urinary tract symptoms other than hematuria.

\section{Morphological Studies}

Tissue Handling. Full thickness bladder samples were taken from the bladder dome and placed immediately in ice cold Krebs solution. Samples for immunofluorescence studies were prefixed in a freshly prepared solution of paraformaldehyde (4\% in phosphate buffered saline [PBS], $\mathrm{pH} 7.4,4^{\circ} \mathrm{C}$ ) for 4 hours, then rinsed and cryoprotected with sucrose $(15 \%$ in PBS, $3 \times 12$ hours, $\left.4^{\circ} \mathrm{C}\right)$. Fixation was carried out within 12 hours to avoid neurotransmitter depletion (Gu et al, 1985). Samples for histochemistry were not prefixed. Samples were frozen in OCT compound (Tissue-Tek; BDH Laboratory Supplies, Poole, United Kingdom) by immersion in isopentane cooled with liquid nitrogen and stored at $-70^{\circ} \mathrm{C}$.

Single- and Double-Label Immunofluorescence. Cryostat sections, $8 \mu \mathrm{m}$ thick, were thaw-mounted onto glass slides, air dried, and incubated for 2 hours in PBS containing Triton X-100 (0.2\%) and bovine serum albumin $(0.1 \%)$. Primary antisera were diluted in the same solution and applied for 24 hours at room temperature, after which excess antibody was removed by washing in PBS $(3 \times 10$ minutes). The second primary antibody for double-labeling studies was then applied for a further 24 hours. Appropriate secondary antibodies (diluted in PBS) were applied for 90 minutes each at room temperature, then washed (PBS, $3 \times 10$ minutes). Texas Red (TR)-conjugated (dilution 1:160) and/or fluorescein isothiocyanate (FITC)-conjugated (dilution 1:80) affinity purified antibodies raised against the source animals of the primary antibodies were used.

Primary antibodies were as follows. From rabbit, PGP 9.5 (working dilution 1:1000; UltraClone, Wellow, Isle of Wight, United Kingdom), NPY (1:400; Peninsula
Laboratories, Belmont, California), gal (1:1500; Peninsula) and HO- 1 and 2 (1:500; StressGen, Victoria, BC, Canada). From guinea pig, VIP (1:640; EuroDiagnostica, Malmö, Sweden), SP (1:1000; EuroDiagnostica). From mouse, CGRP (1:500; EuroDiagnostica), TH (1:1000; Incstar, Stillwater, Minnesota). From rat, SOM (1:500; Euro-Diagnostica). From sheep, n-NOS (1:1500; Euro-Diagnostica). The secondary antibodies used were: swine anti-rabbit (FITC, 1:80; Dako A/S, Glostrup, Denmark), donkey anti-rabbit (TR, 1:160; Jackson ImmunoResearch, West Grove, Pennsylvania), goat anti-guinea pig (FITC, 1:80; Sigma Chemical, St. Louis, Missouri), swine anti-guinea pig (TR, 1:160; Dako), donkey antimouse (TR, 1:160; Jackson), donkey anti-rat (TR, 1:160; Jackson), and donkey anti-sheep (FITC, 1:80; Sigma).

Antiserum specificity control experiments showed no IR in sections incubated in the absence of primary antibody, or in antibody preadsorbed with excess (100 $\mu \mathrm{g} / \mathrm{ml}$ ) of the respective antigen. As cross reactions to antigens with similar amino acid sequences could not be excluded completely, the structures demonstrated are referred to as showing antigen-IR. Nerve fiber frequency (very frequent, frequent, moderate, infrequent, very infrequent, absent) was assessed by two independent observers as compared with PGP 9.5immunolocalized nerve frequency, which was used in controls to determine "very frequent." In double-label immunofluorescence studies, "close apposition" means the two fluorophores had very similar positions but with visible structural differences. "Coinciding profiles" refers to identical structural details. "Colocalization" means the two antigens were present in the same nerve fiber on confocal microscopy.

Confocal Laser Scanning Microscopy. Sections were viewed with a Nikon Eclipse E800 microscope (Nikon, Tokyo, Japan) with a $\times 100$ oil immersion plan apochromat lens. Sequential scanning was performed using excitation wavelengths of 488 and $668 \mathrm{~nm}$ from a Krypton-Argon laser. Immunofluorescence was selected with a $522 \pm 30$ band pass filter for FITC and a $605 \pm 30 \mathrm{~nm}$ band pass filter for Texas Red.

AChE Histochemistry. The Hedreen and Bacon modification (Hedreen et al, 1985) of the KarnovskyRoots stain (Karnovsky and Roots, 1964) was used.

\section{Functional Studies}

Isometric Tension Measurement. Specimens were transferred to Krebs solution at $4^{\circ} \mathrm{C}$ immediately after retrieval. Detrusor strips $(1 \times 1 \times 5 \mathrm{~mm})$ were microsurgically dissected and $5 / 0$ silk ligatures applied to each end. They were mounted at an initial tension of $1 \mathrm{~g}$ in $0.2 \mathrm{ml}$ superfusion organ baths (Brading and Sibley, 1983) and perfused at a flow rate of $1 \mathrm{ml}$ per minute with carboxygenated Krebs solution $\left(0_{2} 97 \%\right.$, $\left.\mathrm{CO}_{2} 3 \%, \mathrm{pH} 7.4\right)$ at $37^{\circ} \mathrm{C}( \pm 0.1)$. One end was attached to a force transducer (Pioden UF1; Harvard Apparatus, Kent, United Kingdom); isometric tension was recorded with an Apple Macintosh PowerBook 1400c computer using Chart software and MacLab 8 
hardware (Ad Instruments Pty, New South Wales, Australia).

Experimental Procedure. After 90 minutes at equilibration, the maximum contractile response of each strip was ascertained with a 10-second application of carbachol $\left(10^{-4} \mathrm{M}\right)$. Forty minutes later, frequencyresponse curves to EFS were constructed by stimulating with a Grass S48 electrical stimulator (Grass Instruments, Quincy, Massachusetts) via recessed platinum ring electrodes. Parameters were set to maximize nerve response, with minimum direct muscle stimulation (5-second pulse trains, 0.05-msec pulse width, $50 \mathrm{~V}$ amplitude, and varying frequencies from 1 to $50 \mathrm{~Hz}$ ) (Sibley, 1984). Graduated increases in the period between stimulations, from 5 minutes at low frequencies (1 to $2.5 \mathrm{~Hz}$ ) to 30 minutes for high frequencies (20 to $50 \mathrm{~Hz}$ ), were applied to avoid desensitization. Atropine resistance was investigated by continuous exposure of the strips to atropine $\left(10^{-6}\right.$ M) for at least 30 minutes before and during repeated stimulation at $30 \mathrm{~Hz}$. Strips were then exposed to tetrodotoxin $\left(5 \times 10^{-7} \mathrm{M}\right)$ with atropine $\left(10^{-6} \mathrm{M}\right)$ to assess the nerve-mediated contribution to any residual contraction at $30 \mathrm{~Hz}$. Finally, strips were blotted on tissue paper and weighed.

\section{Drugs and Solutions}

Carbamylcholine chloride (carbachol), atropine sulfate, and tetrodotoxin (Sigma Chemical, Poole, United Kingdom) were used. Drugs were dissolved in deionized water and diluted in Krebs solution ( $\mathrm{NaCl} 119 \mathrm{~mm}$, $\mathrm{KCl} 4.6 \mathrm{~mm}, \mathrm{CaCl}_{2} 1.5 \mathrm{~mm}, \mathrm{MgCl}_{2} 1.2 \mathrm{~mm}, \mathrm{NaHCO}_{3} 15$ $\mathrm{mm}, \mathrm{NaH}_{2} \mathrm{PO}_{4} 1.2 \mathrm{~mm}$, and glucose $5.5 \mathrm{~mm}$ ). Isopentane for tissue freezing was obtained from $\mathrm{BDH}$, United Kingdom.

\section{Statistics}

Results are expressed as mean \pm SEM. Analysis of variance was used for comparison of parametric data. The Wilcoxon signed ranks test was used for comparison of paired nonparametric data. Statistical significance was inferred at $p<0.05$.

\section{Acknowledgement}

Thanks are due to the Transplant Unit, Churchill Hospital, Oxford, United Kingdom.

\section{References}

Abrams P, Blaivas JG, Stanton SL, Andersen JT, Fowler CJ, Gerstenberg T, and Murray K (1986). Sixth report on the standardisation of terminology of lower urinary tract function. Procedures related to neurophysiological investigations: Electromyography, nerve conduction studies, reflex latencies, evoked potentials and sensory testing. The International Continence Society Committee on Standardisation of Terminology, New York, May 1985. Scand. J Urol Nephrol 20:161164.

Brading AF and Sibley GNA (1983). A superfusion apparatus to study field stimulation of smooth muscle from mammalian urinary bladder. J Physiol (Lond) 334:11-12P.
Brading AF and Turner WH (1994). The unstable bladder: Towards a common mechanism. Br J Urol 73:3-8.

Brading AF and Williams JH (1990). Contractile responses of smooth muscle strips from rat and guinea-pig urinary bladder to transmural stimulation: Effects of atropine and alpha, beta-methylene ATP. Br J Pharmacol 99:493-498.

Brindley GS and Craggs MD (1976). The effect of atropine on the urinary bladder of the baboon and of man. J Physiol 256:55P.

Chapple CR, Milner P, Moss HE, and Burnstock G (1992). Loss of sensory neuropeptides in the obstructed human bladder. Br J Urol 70:373-381.

Charlton RG, Morley AR, Chambers P, and Gillespie JI (1999). Focal changes in nerve, muscle and connective tissue in normal and unstable human bladder. Br J Urol 83:953-960.

Chen TF, Doyle PT, and Ferguson DR (1994). Inhibition in the human urinary bladder by gamma-amino-butyric acid. $\mathrm{Br} \mathrm{J}$ Urol 73:250-255.

Daniel EE, Cowan W, and Daniel VP (1983). Structural bases for neural and myogenic control of human detrusor muscle. Can J Physiol Pharmacol 61:1247-1273.

de Groat WC (1997). A neurologic basis for the overactive bladder. Urology 50 (Suppl. 6A):36-52.

Dixon JS, Jen PYP, and Gosling JA (1998). Immunohistochemical characteristics of human paraganglion cells and sensory corpuscles associated with the urinary bladder. A developmental study in the male fetus, neonate and infant. $J$ Anat 192:407-415.

Elbadawi A, Yalla SV, and Resnick NM (1993). Structural basis of geriatric voiding dysfunction. III. Detrusor overactivity. J Urol 150:1668-1680.

German K, Bedwani J, Davies J, Brading AF, and Stephenson TP (1995). Physiological and morphometric studies into the pathophysiology of detrusor hyperreflexia in neuropathic patients. J Urol 153:1678-1683.

Gilpin SA, Gilpin CJ, Dixon JS, Gosling JA, and Kirby RS (1986). The effect of age on the autonomic innervation of the urinary bladder. Br J Urol 58:378-381.

Gosling JA, Dixon JS, and Humpherson JR (1983). Functional Anatomy of the Urinary Tract. Churchill Livingstone, Edinburgh.

Gu J, Huang WM, and Polak JM (1985). Stability of immunocytochemical reactivity of neuronal substances following delayed fixation. J Neurosci Meth 12:297-302.

Gu J, Restorick JM, Blank MA, Huang WM, Polak JM, Bloom SR, and Mundy AR (1983). Vasoactive intestinal polypeptide in the normal and unstable bladder. Br J Urol 55:645-647.

Harrison SC, Hunnam GR, Farman P, Ferguson DR, and Doyle PT (1987). Bladder instability and denervation in patients with bladder outflow obstruction. Br J Urol 60:519522.

Hedreen JC, Bacon SJ, and Price DL (1985). A modified histochemical technique to visualize acetylcholinesterasecontaining axons. J Histochem Cytochem 33:134-140.

Ho KMT, Ny L, McMurray G, Andersson K-E, A. F. B, and Noble JG (1999). Co-localization of carbon monoxide and nitric oxide synthesizing enzymes in the human urethral sphincter. J Urol 161:1968-1972. 
Iselin CE, Alm P, Schaad NC, Larsson B, Graber P, and Andersson KE (1997). Localization of nitric oxide synthase and haemoxygenase, and functional effects of nitric oxide and carbon monoxide in the pig and human intravesical ureter. Neurourol Urodyn 16:209-227.

Karnovsky MJ and Roots L (1964). A direct coloring thiocholine method for cholinesterase. J Histochem Cytochem 12: 219.

Kinder RB and Mundy AR (1985). Atropine blockade of nerve-mediated stimulation of the human detrusor. $\mathrm{Br} \mathrm{J}$ Urol $57: 418-421$.

Kinder RB and Mundy AR (1987). Pathophysiology of idiopathic detrusor instability and detrusor hyper-reflexia. An in vitro study of human detrusor muscle. Br J Urol 60:509-515.

Kinder RB, Restorick JM, and Mundy AR (1985). Vasoactive intestinal polypeptide in the hyper-reflexic neuropathic bladder. Br J Urol 57:289-291.

Luheshi GN and Zar MA (1990). Presence of non-cholinergic motor transmission in human isolated bladder. J Pharm Pharmacol 42:223-224.

Mills IW (1999). D. M. Thesis: The pathophysiology of detrusor instability and the role of bladder ischaemia in its aetiology. University of Oxford, Oxford, UK.

Mills IW, Greenland JE, McMurray G, McCoy R, Ho KMT, Noble JG, Brading AF (2000). Studies of the pathophysiology of idiopathic detrusor instability: The physiological properties of the detrusor smooth muscle and its pattern of innervation. J Urol 163:646-651.

Moore KH, Gilpin SA, Dixon JS, Richmond DH, and Sutherst JR (1992). Increase in presumptive sensory nerves of the urinary bladder in idiopathic detrusor instability. $\mathrm{Br} \mathrm{J}$ Urol 70:370-372.
Saito M, Kondo A, Kato T, and Levin RM (1993). Response of isolated human neurogenic detrusor smooth muscle to intramural nerve stimulation. $\mathrm{Br} \mathrm{J}$ Urol 72:723-727.

Sibley GN (1984). A comparison of spontaneous and nervemediated activity in bladder muscle from man, pig and rabbit. J Physiol (Lond) 354:431-443.

Sjogren C, Andersson K-E, Husted S, Mattiasson A, and Moller Madsen B (1982). Atropine resistance of transmurally stimulated isolated human bladder muscle. J Urol 128:13681371.

Smet PJ, Edyvane KA, Jonavicius J, and Marshall VR (1994). Distribution of NADPH-diaphorase-positive nerves supplying the human urinary bladder. J Auton Nerv Syst 47:109-113.

Smet PJ, Edyvane KA, Jonavicius J, and Marshall VR (1996). Neuropeptides and neurotransmitter-synthesizing enzymes in intrinsic neurons of the human urinary bladder. Neurocytol 25:112-124.

Smet PJ, Moore KH, and Jonavicius J (1997). Distribution and colocalization of calcitonin gene-related peptide, tachykinins, and vasoactive intestinal peptide in normal and idiopathic unstable human urinary bladder. Lab Invest 77:37-49.

Speakman MJ, Brading AF, Gilpin CJ, Dixon JS, Gilpin SA, and Gosling JA (1987). Bladder outflow obstruction: A cause of denervation supersensitivity. J Urol 138:1461-1466.

Vollerthun R, Hohler B, and Kummer W (1995). Guinea-pig sympathetic postganglionic neurones contain haem oxygenase-2. Neuroreport 7:173-6.

Whiteneck GG, Charlifue SW, Gerhart KA, Lammertse DP, Manley S, Menter RR, and Seedroff KR (1993). Aging with spinal cord injury. New York: Demos. 